\title{
Mutational Landscape of Paired Primary and Synchronous Metastatic Lymph Node in Chemotherapy Naive Gallbladder Cancer
}

\section{Boqiang Fan}

Jiangsu Province Hospital https://orcid.org/0000-0003-4758-1597

\section{Xianfeng Xu}

Nanjing Medical University

Xuehao Wang ( $\nabla$ wangxh@njmu.edu.cn )

Jiangsu Province Hospital and Nanjing Medical University First Affiliated Hospital Department of General Surgery

\section{Research Article}

Keywords: Gallbladder cancer, Genetic mutations, Whole exome sequencing, Next-generation sequencing

Posted Date: August 25th, 2021

DOl: https://doi.org/10.21203/rs.3.rs-815519/v1

License: (a) (1) This work is licensed under a Creative Commons Attribution 4.0 International License. Read Full License

Version of Record: A version of this preprint was published at Molecular Biology Reports on January 5th, 2022. See the published version at https://doi.org/10.1007/s11033-021-06957-y. 


\section{Abstract}

\section{Background:}

Comprehensive genomic analysis of paired primary tumors and their metastatic lesions may provide new insights into the biology of metastatic processes and therefore guide the development of novel strategies for intervention. To date, our knowledge of the genetic divergence and phylogenetic relationships in gallbladder cancer (GBC) is limited.

\section{Methods:}

We performed whole exome sequencing (WES) for 5 patients with primary tumor, metastatic lymph node (LNM) and corresponding normal tissue. Mutations, mutation signatures and copy number variations were analyzed with state-of-art bioinformatics methods. Phylogenetic tree was also generated to infer metastatic pattern.

\section{Results:}

Five driver mutations were detected in these patients. Among which, TP53 was the only shared mutation between primary tumor and LNM. Although tumor mutational burden was comparable between primary tumor and LNM, higher mutation burden was observed in LNM of one patient. Copy number variations (CNVs) burden was higher in LNM than their primary tumor. Phylogenetic analysis indicated both linear and parallel progression of metastasis exist in these patients. TP53 mutation and CNVs were homogenously between primary tumor and LNM.

\section{Conclusions:}

High consistence of genetic landscape were shown between primary tumor and LNM in GBC. However, heterogenicity still exist between primary tumor and LNM in particular patients in term of driver mutation, TMB and CNV burden. Phylogenetic analysis indicated both Linear and parallel progression of metastasis were exist among these patients.

\section{Introduction}

Gallbladder cancer is the most common malignant tumor of the biliary tract worldwide. The prevalence of GBC is highest in Chile, and northern India. Other high risk region include Poland, south Pakistan, Japan and Israel.[1] China is among median rate of incidence. However, based on the population scale, the annual cases are tremendous, which increases the burden of already overwhelming medical resources. Most GBCs, unfortunately, are discovered incidentally at routine cholecystectomy or present as advanced stage disease. Less than $20 \%$ of gallbladder cancer is eligible for potentially curative surgical resection at diagnosis.[2] The overall prognosis of GBC is very poor, despite recent improvement of chemotherapy, molecular targeted therapy and aggressive surgical resection with advances in perioperative care[3]. For advanced stage disease, the 5-year survival is only about 5\%[4]. Regional nodal status and the depth of 
tumor invasion (T status) are the two most important prognostic factors.[4] Genetic landscape studies facilitated by next generation sequencing (NGS) has improved our understanding of this disease in terms of oncogenesis, metastasis and therapeutic. [5-7] However, only primary tumor of GBC was genetic profiled to date.[8-10] Emerging evidence suggest the importance of mutational profile between primary tumor and metastasis.[11-16]

In this study we investigated the primary tumor and their synchronous lymph node metastasis (LNM) with whole exome sequencing (WES) in terms of somatic mutations, mutational signatures[17], and copy number variations (CNV) in order to define if there are distinct mutational landscapes between the two sites.

\section{Materials And Methods}

This study included 5 trios of samples, including primary tumor, metastatic lymph node (LNM), and matched normal tissue of gallbladder. Tumor cell contents from haematoxylin and eosin (H\&E)-stained formalin fixed and paraffin-embedded (FFPE) sections were estimated by a pathologist (W.Y.H).

\section{Whole exome library preparation and sequencing}

For each individual, the genomic DNA of cells from primary tumor, LNM and matched normal tissue sample was sequenced. DNA was extracted from FFPE tissue blocks using QIAGEN QIAAmp DNA extraction kits. The exome of each sample was captured using SureSelect Exon V6 (Agilent Technologies) according to the manufacturer's protocol. The product was quality checked and sequenced with illumina NovaSeq 6000, generating $2 \times 150$ bp paired-end reads.

\section{Sequencing alignment and detection of somatic variants}

The FastQC package (http://www.bioinformatics.babraham.ac.uk/projects/fastqc) was applied to assess the quality-score distribution of the sequencing reads. Paired-end reads were aligned to human reference genome (GRCh37) using the Burrows-Wheeler Aligner (BWA) with default parameters[18]. We then used the Picard-tools 1.76 to fix mate pairs and mark PCR duplicates (http://Picard.Sourceforge.net). The resultant aligned BAM files were then sorted and merged (if needed) using Samtools v0.1.19 [19]. After sorted by Samtools, the reads were subjected to local realignment and recalibration using the Genome Analysis Toolkit (GATK v3.8). Somatic substitutions and indels were called using MuTect2 mode in GATK on the GRCh37 genome with genomic DNA of white blood cells as the germline comparator following the best practices for somatic SNV/indel calling (https://software.broadinstitute.org/gatk/best-practices/). Briefly, the algorithms compared the tumor with the matched normal sample to exclude germline variants. The algorithms applied the following criteria to increase calling reliability: (1) somatic mutations were not found in a panel of normal controls assembled from matched normal tissues,(2) somatic mutations were not located in the segmental duplication region marked by the UCSC browser (http://genome.ucsc.edu/), 
(3) somatic mutations were not found in the 1000 Genomes Project (the Phase III integrated variant set release, across 2, 504 samples) with the same mutation direction. Mutations (SNVs/indels) were annotated with Varcode (https://github.com/openvax/varcode).

\section{Mutational signature analysis}

The R package: Signature Estimation was applied to infer the proportion of each known mutational signature proposed by Alexandrov et al[17]. In total, 30 signatures reported by COSMIC (https://cancer.sanger.ac.uk/cosmic/signatures_v2) were included in the analysis.

\section{Phylogenetic tree construction}

Treeomics v1.7.13, a Bayesian inference model, was applied to reconstruct the phylogeny of the tumor with the multi-region sequencing data for each case[20], which took the numbers of variant reads, read depth, chromosomal coordinates, gene symbol, and substitution pattern into account. Each phylogeny was rooted at the matched patient's normal sample and the leaves represented the primary or metastasis samples.

\section{Estimation of the copy number variations}

The GATK best practices for somatic copy number variations (CNVs) in exomes was applied to detect CNVs from the whole-exome sequencing data (https://software.broadinstitute.org/gatk/best-practices/). We utilized ReCapSeg to estimate the somatic copy number, which is implemented as part of GATK (v4). Briefly, the read counts for each of the exome targets were divided by the total number of reads to generate proportional coverage. A panel of normal (PON) controls was built using proportional coverage from 5 normal samples and each of the tumor samples was compared with the PON, after which tangent normalization was applied. Circular binary segmentation (CBS) was then applied to segment the normalized coverage profiles. Sex chromosomes $(X$ and $Y$ ) were excluded from this analysis. We defined copy number more than 2.5 as gain, and less than 1.5 as loss.

\section{Statistics analysis}

All statistical analyses were performed in R V4.0.2. Comparisons of continuous variables were performed using Mann-Whitney U. All statistical tests were two-tailed and $\mathrm{P}<0.05$ was considered statistically significant.

\section{Results}

Of the 5 patients, mean sequencing depth of the tumor (primary and LNM) and normal tissue were $96 \mathrm{x}$ and $76 x$ respectively (Table $s 1$ ). 


\section{SNV pattern}

In total, we identified 7202 nonsynonymous somatic mutations, including 2808 (range: 400-688) mutations in primary tumor group, and 4394 (range: 239-2344) mutations in LNM group. The main variant type is ins and the main SNV class is C -> T substitution.(Figure S1) We also compare the nonsynonymous somatic mutations between primary tumor group and LNM group (Figure S2), and discovered no significant difference between the two groups.

Driver mutations identified in the 10 tumors by Varcode were TP53, APC, ERBB3, FBXW7 and SMARCB1(figure 1). These driver mutations could be classified as shared (present in both primary and metastatic tumors) and private (present only in the primary or metastatic tumors) for further analysis. TP53 was the only shared mutation and was detected in 2 patients (GBC-1 and GBC-2). The mutation pattern of TP53 in GBC-1 was in frame deletion at the same locus (17:7577514-7577517, GTGA->G). Similar mutation was reported in COSMIC (17:7577514-7577516, p.T256del , Deletion - In frame, and 17:7577515-7577516, p.L257Gfs*6, Deletion - Frameshift). And in GBC-2, it was missense mutation at same locus (17:7577539, G->A), which was not reported in COSMIC. Private mutation was identified in 3 patients either additional to shared mutation (GBC-1 and GBC-2) or merely in primary tumor (GBC-5). There were no known driver mutation in GBC-3 and GBC-4, which may imply other mechanism of oncogenesis [21, 22]. Theoretically, three driver genes are required to convert a normal cell to a cancer cell in solid tumors, and an average of approximately four driver genes were actually harbored per tumor[23]. In this study, none of the 10 tumor samples were recognized more than 2 known driver mutations. This result implies not all driver mutations could be recognized currently.

We also calculated tumor mutational burden (TMB), the median TMB was 5.82 per Mb and 5.49 per Mb in primary tumor group and LNM group respectively. Median non-silent TMB was 1.84 per Mb and 1.80 per $\mathrm{Mb}$ in primary tumor group and LNM group respectively (Figure S3). Significant higher TMB (24.65 per MB) was found in the LNM lesion of GBC-3. There were no significant difference between the two groups.

\section{Mutational Signatures}

Annotated with COSMIC V2, major signatures are similar in both groups. Signature 3, 1, 6, 12, 11, 22, 23, and 7 were detected in primary group (Figure S4). Signature 1 is age related represent a large numbers of $\mathrm{C}>\mathrm{T}$ mutations. Signature 3 is strongly associated with germline and somatic BRCA1 and BRCA2 mutations in breast, pancreatic, and ovarian cancers. In pancreatic cancer, responders to platinum therapy usually exhibit signature 3 mutations[24]. These patients did not exhibit BRCA1 and BRCA2 mutations, although BRCA mutations have been reported in GBC patients[25]. Additionally, signature 9 was also enriched in LNM group. This signature is characterized by a pattern of mutations that has been attributed to polymerase $\eta$, which is implicated with the activity of Activation-induced cytidine deaminase (AID) during somatic hypermutation. Signature 12, 22 and 23 were also enriched in both groups (20\% and $28 \%$ ) implies there were some extent of similarity between GBC and liver cancer. 


\section{Phylogenetic tree}

Based on SNVs, we identified two types of phylogenetic tree with Treeomics, according to previous classification of driver mutations as shared or private. The length of trunk and branch was corresponding to nonsynonymous somatic mutation numbers (Figure 2). All known driver mutations were mapped on the tree. Since the VAF (Variant allele frequencies, calculated by total reads at the position carrying the variant/read depth at the position) of TP53 in GBC-2 was very low (1/36), Treeomics judged this mutation was unreliable. We then re-checked this mutation and manually included into analysis, since this mutation is shared in both primary tumor and LNM, and mutate at same locus (17:7577539, G->A). Linear or parallel progression models of metastasis were both identified in other tumors[26, 27]. The trees of our patients inferred both pattern of metastasis were existed. GBC- 1 and GBC-2 inferred a linear pattern and GBC-5 inferred a parallel patten.

\section{CNV}

We then analyzed copy number variations. Compared to reported $\operatorname{CNVs}[9,28]$, there were more losses than gains. In total, there were 3 gains and 11 losses in the samples (Figure 3). Losses of 8p23.3 $9 q 21.11 \otimes 14 q 32.13 \otimes 16 q 23.1$ were detected in several samples. Some CNVs were detected in both primary tumor and LNM. In GBC-1, gain of 17q21.1, and losses of 9q21.11 and 8p23.3 were detected. In GBC-2, 9q21.11 loss was detected. Losses of 8p23.3, 9q21.11 and 15q23.1 were detected in GBC-3.

Apart from the reported CNVs, we newly identified large segments alterations in both primary tumor and LNM in 2 patients. In GBC-5, the gain proportion was 0.99 and 0.75 in chromosome 7, and 0.69 and 0.65 in chromosome 20 for the primary tumor and LNM, respectively. In GBC-3, the loss proportion was 0.75 both in primary tumor and LNM in chromosome 10. Thus, whole arm gain and loss could be inferred in these two patients.

We further calculated CNV burden by dividing the copy numbers of loss or gain by all copy numbers founded with GATK (Figure S6). Median CNV loss burden was 0.013 and 0.043 in primary tumor and LNM respectively. Median CNV gain burden was 0.0009 and 0.0671 in primary tumor and LNM respectively. Though, there was no statistical significance in general due to limited patient number, higher CNV burden, either gain or loss was observed in LNM than their primary tumor in 3 patients.

\section{Discussion}

Despite recent improvement of chemotherapy, molecular targeted therapy and aggressive surgical resection with advances in perioperative care has markedly improved outcomes, the prognosis of GBC is still very poor. Molecular profiling of the primary tumor has significantly improved our understanding of this disease in terms of oncogenesis, prevention and treatment [5, 29-33]. However, the metastasis comprises a major obstacle to treatment and long-term survival. It is believed that a better understanding of mutational landscape and evolutionary pattern between primary tumor and metastasis would have 
profound clinical implications, as such findings could ultimately result in novel strategies to prevent and control metastases[12, 13,34-36], especially in the era of personalized medicine[5, 6, 37].

Although, a variety of driver mutations have been reported in GBC[38], TP53 is the most frequent one in current study (20\%). The mutation patterns of LNM is homogenous with their primary tumors, which is consistent with studies on other tumors, including breast, colorectal, and hepatocellular cancer $[16,35,39$, 40]. A recent analysis of genetic heterogeneity in untreated cancers also found $100 \%$ of the driver mutations were homogeneous among metastases from the same primary tumor, indicating the high homogeneity of functional driver mutations between paired primary and metastatic lesions[34]. These findings together reached an opinion of using the sequencing data of primary tumors to guide the treatment of metastatic lesions. However, this is not always the rule. There were 4 mutations only detected in either primary or LNM. In the view of personalized therapeutics, the different mutations may imply particular clinical significance.

TMB is increasingly accepted as a biomarker for immunotherapy. High TMB is correlated with good response. Although the definition of "TMB-high" is not consistent among literature, the cutoff value is usually set to 10 [41] or 20 [42-44]. In this study, TMB is generally less than 10 both in primary tumor and LNM ( 5.92/5.48 mutations per MB). This is consist with previous studies[8, 45]. TMB is generally considered as a result of DNA damage repair deficiency, and correlates with mismatch repair genes and microsatellite instability (MSI).[46] The only TMB high sample (LNM in GBC-3, TMB=24.65 per MB) showed no such genetic feature. Further analysis of mutation signatures showed signature 9 may contribute to this. Although in most patients there is an opinion that a single biopsy of a primary tumor captures the information necessary for therapeutic choices about the treatment of extant or presumptive metastases[34], in terms of TMB, metastasis and primary tumor may not represent mutually. Differences of TMB were also detected in other tumors. Pulmonary metastasis of osteosarcoma[47] and peritoneal metastasis of colorectal cancer[48] indicated higher mutation burden than their primary tumor. If only the primary tumor was examined, the TMB of the metastasis would be underestimated, leading to a misjudgment of the choice of immunotherapy. In a practical point of view, when immunotherapy is to be considered, at least the significant lesion should be examined.

Since CNV is an important indicator of chromosomal instability (CIN) and previous publications suggested that CIN is associated with metastasis.[49-51] Advanced tumors typically contain frequent gains and losses of focal genomic regions, chromosome-arms, and whole chromosomes.[34] Our analysis of CNV in the two groups showed consistent alterations in primary tumor and LNM. Even chromosome arm aneuploidies were consistent between primary tumor and LNM. A study of breast cancer also showed ERBB2/HER2 amplification and/or mutation frequency was consistent between primary tumor and metastases[52]. Our pilot analysis of CNV burden exhibited difference between primary tumor and LNM, though there is no related study. The clinical significance is to be tested.

Finally we built the phylogenetic tree to infer the evolution of the metastasis. Our patients showed both linear and parallel progression of metastasis. Studies of other tumor also inferred both models of 
metastasis could co-exist[26, 27]. Due to the small sample size, we can not conclude which model is dominant.

There are several Limitations of the study. Firstly, only 5 patients were included in this study, and this is surely underpower our analysis. Collecting larger scale cases is warranted to further validate our current finding or may generate new findings. Secondly, only metastatic lymph node was included, other metastases eg. liver or lung metastasis were not included due to scarce of samples. Since patients with such metastasis were usually not surgical candidates. Thirdly, all the 5 patients were at late stage (stage III or stage IV). Our results of homogeneous CNV and driver mutation between primary tumor and LNM imply early metastasis with the evidences of. Recent evidence suggested metastasis could occur at early stage $[36,53]$ and even 7\% T1a tumor may have concomitant LNM[54]. Both indicate further analyses to generate robust data for this hypothesis.

Taken together, our study with WES showed high consistence of genetic landscape between primary tumor and LNM in GBC. However, heterogenicity still exist between primary tumor and LNM in particular patients in term of driver mutation, TMB and CNV burden. These differences may have clinical significances.

\section{Declarations}

Funding: There is no funding for current work.

Conflicts of interest/Competing interests: There is no conflicts of interest for all authors.

Availability of data and material: All raw data is available, and is ready to share.

Code availability: All the software used in this manuscript is available online, and there is no custom code.

Authors' contributions: XF Xu and BQ Fan performed WES, critical data analyses, interpretation of results and wrote the manuscript. XF Xu performed DNA extraction, analyzed the data and carried out statistical analyses. BQ Fan contributed samples and analyzed clinical data. BQ Fan and XH Wang designed, implemented the study, wrote and critically reviewed the manuscript. This is to confirm that all authors read and approved the final manuscript.

Additional declarations for articles in life science journals that report the results of studies involving humans and/or animals

Ethics approval: Since this is an retrospective diagnostic study, no previous agreement from our Ethical Committee was obtained. 
Consent to participate: not applicable.

\section{Consent for publication: not applicable.}

\section{References}

1. Bray, F.I., et al., Global cancer statistics 2018: GLOBOCAN estimates of incidence and mortality worldwide for 36 cancers in 185 countries. CA: A Cancer Journal for Clinicians, 2018. 68(6): p. 394424.

2. Schmidt, M.A., L. Marcano-Bonilla, and L.R. Roberts, Gallbladder cancer: epidemiology and genetic risk associations. Chinese Clinical Oncology, 2019. 8(4): p. 2.

3. Lai, C.H.E. and W.Y. Lau, Gallbladder cancer - A comprehensive review. Surgeon-journal of The Royal Colleges of Surgeons of Edinburgh and Ireland, 2008. 6(2): p. 101-110.

4. Kanthan, R., et al., Gallbladder Cancer in the 21st Century. J Oncol, 2015. 2015: p. 967472.

5. Lin, J., et al., Precision oncology for gallbladder cancer: insights from genetic alterations and clinical practice. Annals of Translational Medicine, 2019. 7(18): p. 44.

6. Haber, P.K. and D. Sia, Translating cancer genomics for precision oncology in biliary tract cancers. Discov Med, 2019. 28(155): p. 255-265.

7. Valle, J.W., et al., New Horizons for Precision Medicine in Biliary Tract Cancers. Cancer Discovery, 2017. 7(9): p. 943-962.

8. Li, M., et al., Whole-exome and targeted gene sequencing of gallbladder carcinoma identifies recurrent mutations in the ErbB pathway. Nat Genet, 2014. 46(8): p. 872-6.

9. Nakamura, H., et al., Genomic spectra of biliary tract cancer. Nat Genet, 2015. 47(9): p. 1003-10.

10. Jiao, Y., et al., Exome sequencing identifies frequent inactivating mutations in BAP1, ARID1A and PBRM1 in intrahepatic cholangiocarcinomas. Nat Genet, 2013. 45(12): p. 1470-1473.

11. Li, L., et al., Genetic Heterogeneity Between Paired Primary and Brain Metastases in Lung Adenocarcinoma. Clin Med Insights Oncol, 2020. 14: p. 1179554920947335.

12. Jiang, T., et al., Mutational landscape and evolutionary pattern of liver and brain metastasis in lung adenocarcinoma. J Thorac Oncol, 2020.

13. Li, C., et al., Mutational landscape of primary, metastatic, and recurrent ovarian cancer reveals c-MYC gains as potential target for BET inhibitors. Proc Natl Acad Sci U S A, 2019. 116(2): p. 619-624.

14. Ng, C.K.Y., et al., Genetic Heterogeneity in Therapy-Naïve Synchronous Primary Breast Cancers and Their Metastases. Clin Cancer Res, 2017. 23(15): p. 4402-4415.

15. Dai, W., et al., Whole-exome sequencing reveals critical genes underlying metastasis in oesophageal squamous cell carcinoma. J Pathol, 2017. 242(4): p. 500-510.

16. Ouyang, L., et al., Whole-genome sequencing of matched primary and metastatic hepatocellular carcinomas. BMC Med Genomics, 2014. 7: p. 2. 
17. Alexandrov, L.B., et al., Clock-like mutational processes in human somatic cells. Nat Genet, 2015. 47(12): p. 1402-7.

18. Li, H. and R. Durbin, Fast and accurate short read alignment with Burrows-Wheeler transform. Bioinformatics, 2009. 25(14): p. 1754-60.

19. Li, H., et al., The Sequence Alignment/Map format and SAMtools. Bioinformatics, 2009. 25(16): p. 2078-9.

20. Reiter, J.G., et al., Reconstructing metastatic seeding patterns of human cancers. Nat Commun, 2017. 8: p. 14114.

21. Martincorena, I. and P.J. Campbell, Somatic mutation in cancer and normal cells. Science, 2015. 349(6255): p. 1483-9.

22. Poulos, R.C. and J.W.H. Wong, Finding cancer driver mutations in the era of big data research. Biophys Rev, 2019. 11(1): p. 21-29.

23. Martincorena, I., et al., Universal Patterns of Selection in Cancer and Somatic Tissues. Cell, 2017. 171(5): p. 1029-1041.e21.

24. Waddell, N., et al., Whole genomes redefine the mutational landscape of pancreatic cancer. Nature, 2015. 518(7540): p. 495-501.

25. Gerlinger, M., et al., Genomic architecture and evolution of clear cell renal cell carcinomas defined by multiregion sequencing. Nat Genet, 2014. 46(3): p. 225-233.

26. Turajlic, S., N. McGranahan, and C. Swanton, Inferring mutational timing and reconstructing tumour evolutionary histories. Biochim Biophys Acta, 2015. 1855(2): p. 264-75.

27. Hong, W.S., M. Shpak, and J.P. Townsend, Inferring the Origin of Metastases from Cancer Phylogenies. Cancer Res, 2015. 75(19): p. 4021-5.

28. Wardell, C.P., et al., Genomic characterization of biliary tract cancers identifies driver genes and predisposing mutations. J Hepatol, 2018. 68(5): p. 959-969.

29. Lamarca, A., et al., Molecular targeted therapies: Ready for "prime time" in biliary tract cancer. J Hepatol, 2020. 73(1): p. 170-185.

30. Athauda, A., et al., Broadening the therapeutic horizon of advanced biliary tract cancer through molecular characterisation. Cancer Treat Rev, 2020. 86: p. 101998.

31. Goldstein, D., C. Lemech, and J. Valle, New molecular and immunotherapeutic approaches in biliary cancer. ESMO Open, 2017. 2(Suppl 1): p. e000152.

32. Javle, M., et al., Biliary cancer: Utility of next-generation sequencing for clinical management. Cancer, 2016. 122(24): p. 3838-3847.

33. Xu, S., et al., Genome-wide CRISPR screen identifies ELP5 as a determinant of gemcitabine sensitivity in gallbladder cancer. Nat Commun, 2019. 10(1): p. 5492.

34. Reiter, J.G., et al., An analysis of genetic heterogeneity in untreated cancers. Nat Rev Cancer, 2019. 19(11): p. 639-650. 
35. Wei, Q., et al., Multiregion whole-exome sequencing of matched primary and metastatic tumors revealed genomic heterogeneity and suggested polyclonal seeding in colorectal cancer metastasis. Ann Oncol, 2017. 28(9): p. 2135-2141.

36. Wan, L., K. Pantel, and Y. Kang, Tumor metastasis: moving new biological insights into the clinic. Nat Med, 2013. 19(11): p. 1450-64.

37. Lamarca, A., et al., Current standards and future perspectives in adjuvant treatment for biliary tract cancers. Cancer Treatment Reviews, 2020. 84: p. 101936.

38. Mehrotra, R., et al., Genetic landscape of gallbladder cancer: Global overview. Mutation Researchreviews in Mutation Research, 2018. 778: p. 61-71.

39. Sylvester, B.E. and E. Vakiani, Tumor evolution and intratumor heterogeneity in colorectal carcinoma: insights from comparative genomic profiling of primary tumors and matched metastases. $\mathrm{J}$ Gastrointest Oncol, 2015. 6(6): p. 668-75.

40. Lee, S.Y., et al., Comparative genomic analysis of primary and synchronous metastatic colorectal cancers. PLoS One, 2014. 9(3): p. e90459.

41. Barroso-Sousa, R., et al., Tumor Mutational Burden and PTEN Alterations as Molecular Correlates of Response to PD-1/L1 Blockade in Metastatic Triple-Negative Breast Cancer. Clin Cancer Res, 2020. 26(11): p. 2565-2572.

42. Goodman, A.M., et al., Tumor Mutational Burden as an Independent Predictor of Response to Immunotherapy in Diverse Cancers. Mol Cancer Ther, 2017. 16(11): p. 2598-2608.

43. McNamara, M.G., et al., Impact of high tumor mutational burden in solid tumors and challenges for biomarker application. Cancer Treat Rev, 2020. 89: p. 102084.

44. Hatakeyama, K., et al., Tumor mutational burden analysis of 2,000 Japanese cancer genomes using whole exome and targeted gene panel sequencing. Biomed Res, 2018. 39(3): p. 159-167.

45. Nakamura, H., et al., Genomic spectra of biliary tract cancer. Nature Genetics, 2015. 47(9): p. 10031010.

46. Chalmers, Z.R., et al., Analysis of 100,000 human cancer genomes reveals the landscape of tumor mutational burden. Genome Med, 2017. 9(1): p. 34.

47. Wang, D., et al., Multiregion Sequencing Reveals the Genetic Heterogeneity and Evolutionary History of Osteosarcoma and Matched Pulmonary Metastases. Cancer Res, 2019. 79(1): p. 7-20.

48. Hallam, S., et al., The transition from primary colorectal cancer to isolated peritoneal malignancy is associated with an increased tumour mutational burden. Sci Rep, 2020. 10(1): p. 18900.

49. Robinson, D., et al., Integrative clinical genomics of advanced prostate cancer. Cell, 2015. 161(5): p. 1215-1228.

50. Campbell, P.J., et al., The patterns and dynamics of genomic instability in metastatic pancreatic cancer. Nature, 2010. 467(7319): p. 1109-13.

51. Yates, L.R., et al., Subclonal diversification of primary breast cancer revealed by multiregion sequencing. Nat Med, 2015. 21(7): p. 751-9. 
52. Priedigkeit, N., et al., Intrinsic Subtype Switching and Acquired ERBB2/HER2 Amplifications and Mutations in Breast Cancer Brain Metastases. JAMA Oncol, 2017. 3(5): p. 666-671.

53. Hosseini, H., et al., Early dissemination seeds metastasis in breast cancer. Nature, 2016. 540(7634): p. 552-558.

54. Yang, Y., et al., A predictive nomogram for lymph node metastasis of incidental gallbladder cancer: a SEER population-based study. BMC Cancer, 2020. 20(1): p. 828.

\section{Figures}

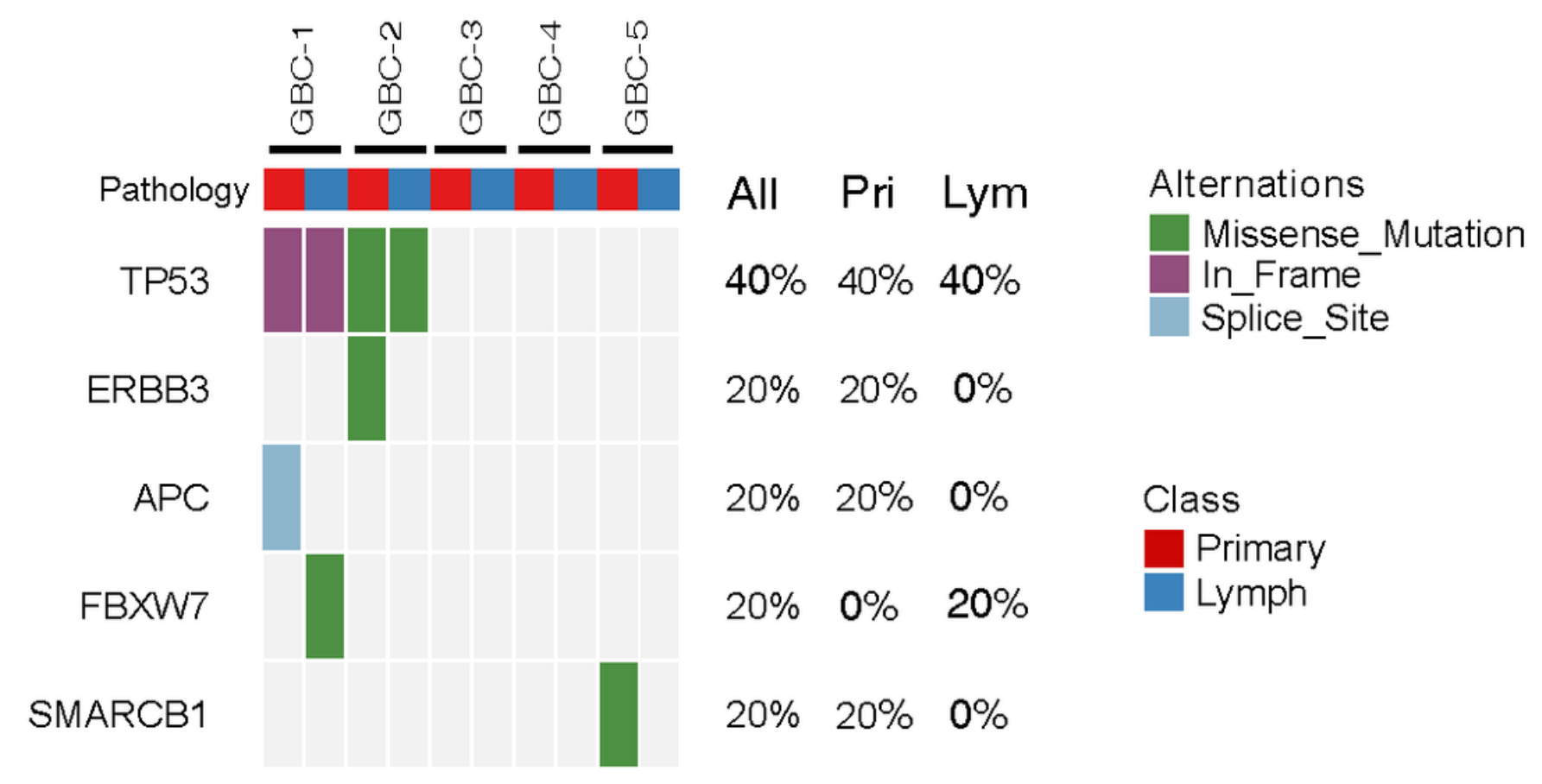

\section{Figure 1}

Driver mutations identified in the 10 tumors by Varcode were TP53, APC, ERBB3, FBXW7 and SMARCB1 

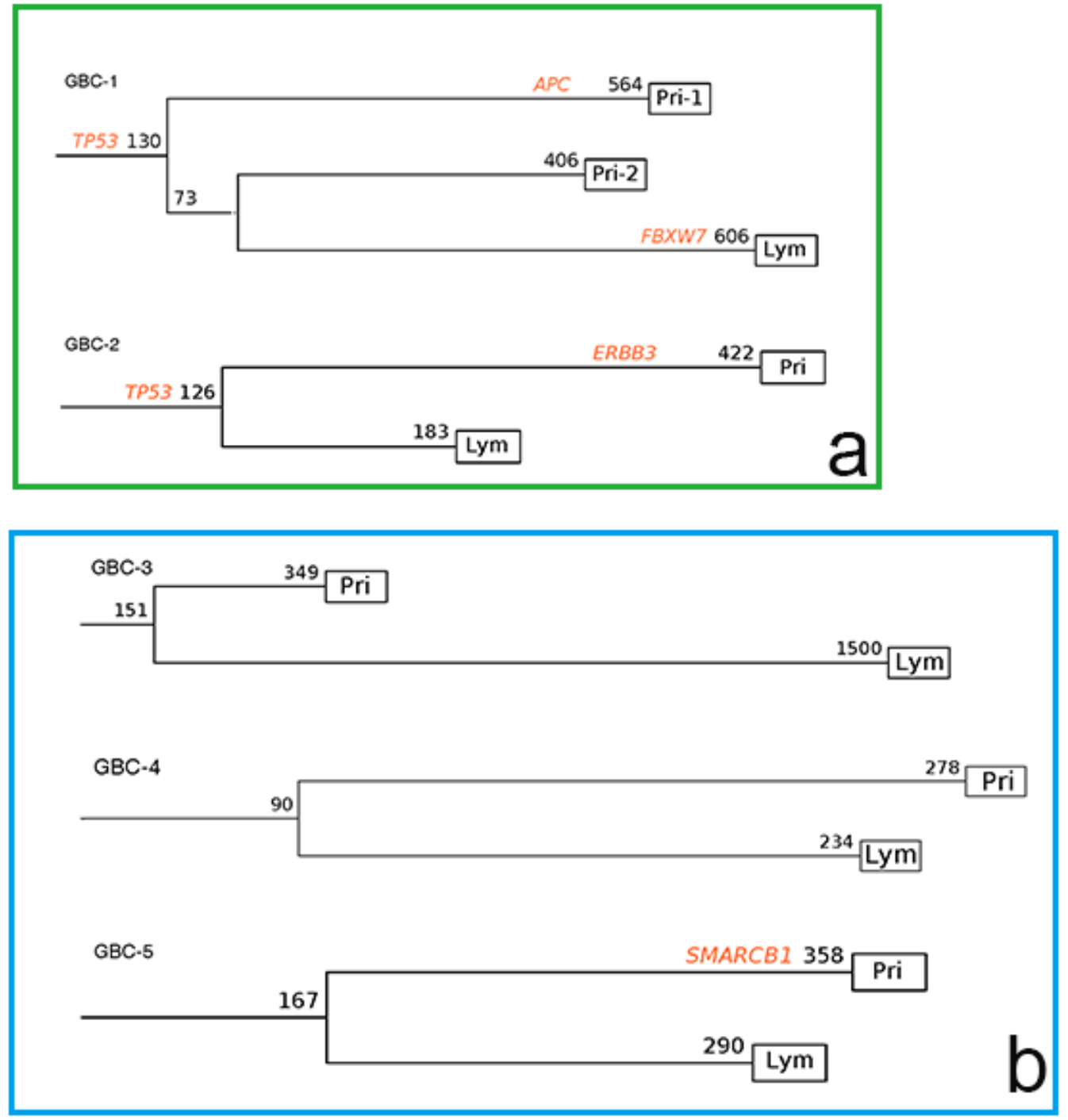

Figure 2

Based on SNVs, we identified two types of phylogenetic tree with Treeomics, according to previous classification of driver mutations as shared or private. The length of trunk and branch was corresponding to nonsynonymous somatic mutation numbers

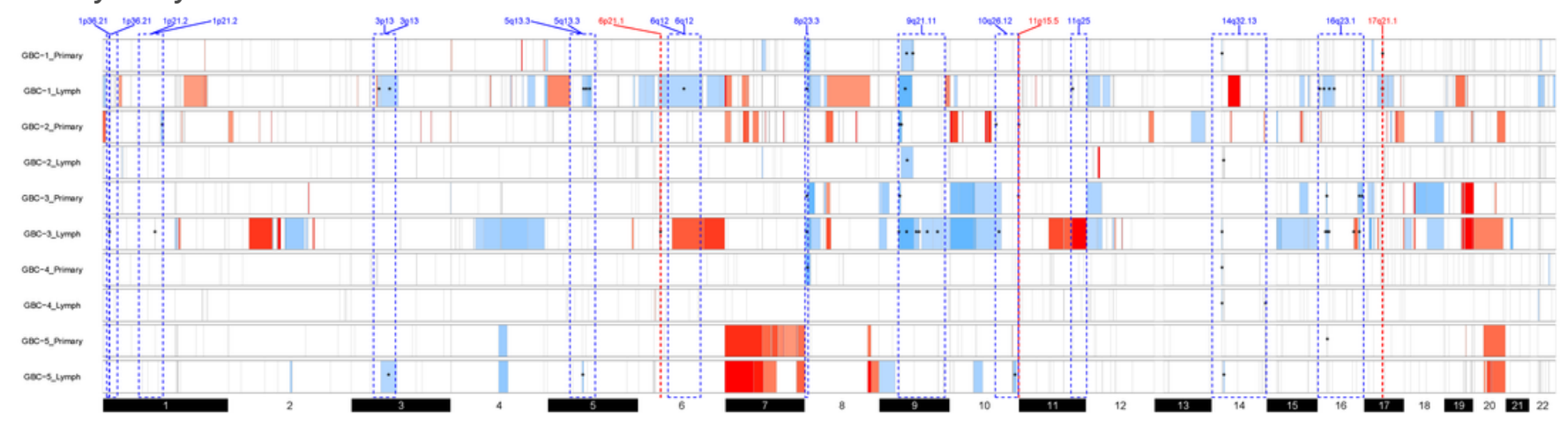

Figure 3 
We then analyzed copy number variations. Compared to reported CNVs[9,28], there were more losses than gains. In total, there were 3 gains and 11 losses in the samples

\section{Supplementary Files}

This is a list of supplementary files associated with this preprint. Click to download.

- FigureS1.tif

- Figures2.tif

- Figures2.tiff

- FigureS3a.tiff

- FigureS3b.tif

- FigureS4a.tif

- FigureS4b.tif

- FigureS5a.CNVGAINBurdenALL.tif

- FigureS5b.CNVGAINallsample.tif

- FigureS5c.CNVLOSSBurdenALL.tif

- FigureS5d.CNVLOSSallsample.tif

- TableS1.xlsx 